\title{
EDITORIAL
}

\section{Lipid Accumulation Product (LAP) Index as Predictor Tool for Metabolic Syndrome}

Metabolic syndrome (MetS) is now considered as an emerging public health issue due to increased mortality and multiple morbidities across the globe. It can be defined as constellation of various conditions which can occur in combination leading to increased risk of coronary artery diseases, stroke, diabetes mellitus (DM), fatty liver and other adverse health conditions. Though there is variation in diagnostic criteria decided by different international bodies like NCEP ATP-III, IDF, WHO; features like increased waist circumference (WC), raised triglyceride (TG) are common component to all criteria $^{1,2}$. Each component has been revealed as an independent risk for cardiovascular disease (CVD) events or DM incidence but three or more combination of factors with diagnosis of MetS increase the chance of developing severe CVD events and DM in future.

If we look at the evidences of different studies done globally which revealed an increased trend of prevalence and incidence of MetS ${ }^{2,3}$. One of the recent study explored increased trend of MetS from $32.5 \%$ to $36.9 \%$ in US population from 2011 to $2016^{4}$. Trend varies with age, sex and ethnicity. This study showed in women from $31.7 \%$ to $36.6 \%$, adult male (20-39 years) from $16.2 \%$ to $21.3 \%$. MetS was found to have increased trend from $19.9 \%$ to $26.2 \%$ in Asian ethnicity and $32.9 \%$ to $40.4 \%$ in Hispanic. one in five in young, half of adult with age 60 and above are found to have MetS. Prevalence is found to be raised with increasing age $24 \%$ in adult over 20 years and $44 \%$ by age 50 years. Prevalence of MetS varies not only due to different ethnic origin but also by using different tools of standard criteria fixed by different international bodies ${ }^{2-5}$.

Considering the etiopathology of MetS, insulin resistance (IR) plays central role whatever the genetic and family history are there. Expression of the disease will be revealed due to unhealthy food habit and less physical activities ${ }^{2,5,6}$. These interaction leads to overweight and obesity, particularly central or visceral fat accumulation, hence IR and subsequent MetS. Specific distribution and functional changes of visceral fat cell are thought to play role. Inflammatory cytokines and adipokines and leptin released from adipocyte are the postulated mechanism for IR. Until recent surrogate markers like BMI, WC, IR are studied and practiced to predict MetS ${ }^{5-7}$. Positive correlation were found for increased IR as well as MetS with high BMI, increased WC and hight-waist ratio ${ }^{6-8}$.

In 1905, Khan HS proposed lipid accumulation product (LAP) as a new index for better predictive value for MetS using WC and serum TG together based on population-based study NHANES III ${ }^{9}$. LAP is inexpensive tool which reflect lipid accumulation. IR, waist circumference, atherogenic lipid, endothelial dysfunction are postulated as central role in MetS. First two are the prime factors in the pathogenesis. Obesity, particularly visceral obesity is closely related to IR. IR, even in non-obese may increase the tendency to develop MetS and CVD. Expensive, complex, time consuming euglycemic hyper insulinemic clamp study is considered as the gold standard to evaluate IR but simple HOMAIR is used in epidemiology survey. Some clinical parameter like BMI, waist circumference are usually used to identify IR but such indices are not so much satisfactory, can not distinguish lean and fat tissue, nor reflect lipid accumulation nor distribution nor functional abnormalities. Measurement of WC can not reflect sufficiently visceral fat or even are unable to distinguish subcutaneous adipose tissue from visceral adipose tissue $^{7,8,10}$. LAP index were tested on different populations, non-diabetes population, healthy population as well as PCOS. LAP were revealed as simple, accurate, stronger predictive index to recognize IR, MetS and CVD risk in non-diabetic population as well as healthy people of different ethnicity. It has effective index with higher sensitivity and specificity than BMI, WC predicting $\mathrm{CVD}^{10-14}$. A recent paper published in current issue of the Journal of Bangladesh College of Physicians and Surgeons (JBCPS) evaluating sensitivity, specificity, positive predictive value and negative predictive value of LAP in detection of MetS 
in male study subjects were $70.45 \%, 78.57 \%, 72.09 \%$ and $77.19 \%$ respectively and in females these values were $20.45 \%, 96.43 \%, 81.82 \%$ and $60.67 \%$ respectively, highlighting its utility in Bangladeshi population.

ATTICA, a prospective Caucasian population based cohort study, showed incidence of CVD in 317 cases $(15 \%)$ in 3042 adults after 10 years follow up with baseline LAP. These findings support a positive association between LAP and long term CVD incidence ${ }^{15}$. More over LAP index was found to predict the incidence of CVD better than BMI, WC, waist -hip, waist-height ratio in other studies ${ }^{7,15}$. Similarly, utilizing clinical and laboratory finding together visceral adiposity Index (VAI) can be used as a novel marker of fat distribution and function as is found by LAPindex. Exploring as moderate to high accuracy with high sensitivity and specificity we can also consider VAI as low cost and easily available screening marker of $\mathrm{MetS}^{16}$.

In conclusion, MetS is increasing as a silent epidemic globally, as well as, in Bangladesh due to rapid economic growth and life style changes. CVD and DM are running after as a consequence. Early, rapid with high accuracy and low-cost screening predictive index like LAP can help to identify the risk component and to take preventive strategies.

(J Bangladesh Coll Phys Surg 2022; 40: 1-2) DOI: https://doi.org/10.3329/jbcps.v40i1.57050

\section{Prof. Md. Faruque Pathan}

Professor

Department of Endocrinology and Metabolism, BIRDEM General Hospital and

Director, BIRDEM Academy, Dhaka, Bangladesh

Email: pathan279@yahoo.com

\section{References:}

1. Genser L, Rossario J, Mariolo C, Castagneto-Gissey L, Panagiotopoulos S, Rubino F. Obesity, type 2 Diabetes and Metabolic Syndrome. Sur Clin N Am 2016;96:681-701.

2. Saklayen MG. The Global Epidemic of the Metabolic Syndrome. Current Hypertension Reports 2018;20:12.

3. Ranasinghe P, Mathangasinghe Y, Hills A, Misra A. Prevalence and trends of metabolic syndrome among adults in the Asia-Pacific region: a systemic review. BMC Public Health 2017;17:101.
4. Hirode G, Wong RJ. Trends in the prevalence of Metabolic Syndrome in the United States 2011-2016. JAMA 2020:303(24);2526-8.

5. Misra A, Khurana L. Obesity and the metabolic syndrome in developing countries. J Clin Endocrinol Metab 2008;93(11):s9-s30.

6. Depres J-P, Lemieux I, Bergeron J, Pibarot P, Mathieu P, Larose E, et al. Abdominal Obesity and the Metabolic Syndrome: Contribution to Global Cardiometabolic Risk. Arteroscler Thromb Vasc Biol 2008;28:1039-49.

7. Bener A, Yousufzai MT, Darwish S, Al-Hamaq A.O.A.A, Nasrulla EA, Ghani MA. Obesity Index That Better Predict Metabolic Syndrome: Body Mass Index, Waist Circumference, Waist Hip Ratio, or Waist Height Ratio. J of Obesity 2013.dx.doi.org/10.1155/2013/269038

8. Wong JC, O Neill S, Beck BR, Ferwood MA, Khoo SK. Comparison of obesity and metabolic syndrome using fat mass index, body mass index, percentage body fat. PLos ONE 2021;16(1):e0245436.

9. Khan HS. The lipid accumulation product performs better than the body index for recognizing cardiovascular risk: a population-based comparison. BMC Cardivasc Disord $2005 ; 5: 26$

10. Khan HS. The Lipid Accumulation Product Is Better Than BMI for Identifying Diabetes. Diabetes Care 2006;29(1):151-3.

11. Zia RL, Zhang S, Gong L, Ren W, Wang Z, Li Q. Lipid accumulation product is a powerful index for recognizing insulin resistance in non-diabetic individuals. Europ J Clin Nutri 2012;66:1035-8.

12. Taverna MJ, Larrad MTM, Frechtel GD, Rios MS. Lipid accumulation product: a powerful marker of metabolic syndrome in healthy population. Europ J Endocrinol 2011;164:559-67.

13. Bermudez V, Salazar Z, Fuenmayor J, Nava M, Ortega A, Duran P, et al. Lipid Accumulation Product Is more Related to Insulin Resistance than the Visceral Adiposity Index in the Maracaibo City Population, Venezuela. J of Obesity 2021.doi.org/10.1155/2021/5514901

14. Wiltgen D, Benedetto IG, Mastella LS, Spritzer PM. Lipid accumulation product index: a reliable marker of cardiovascular risk in polycystic ovary syndrome. Human Reproduction 2009;24(7):1726-31.

15. Kyrou I, Panagiotakas DB, Kouli GM, Georgusopoulou E, Chrysohoou C, Tsigos C, et al. Lipid accumulation product in relation to 10 year cardiovascular disease incidence in Caucasian aduts: The ATTICA Study. Atherosclerosis 2018;279:1016

16. Bijari M, Jangjoo S, Emami N, Raji S, Mottaghi M, Moallem $\mathrm{R}$, et al. The Accuracy of Visceral Adiposity Index for the Screening of Metabolic Syndrome: A Systemic Review and Meta-Analysis. Intern J Endocrinol 2021.doi.org/10.1155/ 2021/6684627 